\title{
A Beam Breakup Instability in a Recirculating Linac Caused by a Quadrupole Mode*
}

\author{
Byung C. Yunn ${ }^{\dagger}$ \\ Thomas Jefferson National Accelerator Facility, Newport News, VA 23606, USA
}

\begin{abstract}
Following the successful demonstration of energy recovery in a recirculating linac with superconducting cavities at the Jefferson Lab FEL[1], several ambitious electron accelerator projects have recently been proposed or are in study for either a light source or a collider based on this novel technology. These projects all intend to utilize a high quality linac electron beam generated with an average beam current typically in the range of 100's of $\mathrm{mA}$. As is well known, a recirculating linac suffers from a beam breakup instability of a regenerative type caused by a Higher Order Mode (HOM) in an accelerating cavity, which can have a very high $\mathrm{Q}$ (quality factor) when superconducting. The instability can degrade the beam quality significantly. We investigate one such beam breakup instability in a recirculating linac that could arise as a result of high $\mathrm{Q}$ quadrupole modes excited in the cavity. In a simple model we derive a relation which allows one to deduce the upper limit on tolerable $\mathrm{Q}$ values for such modes. The results should provide a useful guide in setting the HOM damping requirement among others.
\end{abstract}

\section{INTRODUCTION}

A recirculating linac with an energy recovery is especially suitable for a high beam power accelerator project. While preserving the outstanding beam quality which the linac can provide it shows a way to substantially reduce costs involving RF power generation and consumption. Also significantly less are environmental problems and high cost associated with the construction of high power beam dumps necessary for such a high beam power project.

In order to take a full advantage of energy recovery feature superconducting cavities are preferred for acceleration in such a linac. Extremely high Q values for HOMs in the order of $10^{9}$ to $10^{10}$ are commonly observed for a superconducting RF (SRF) cavity when not damped. As is well known damping of HOMs in a SRF cavity is a major research field in developing SRF technology for the application in accelerator projects.

It is also well acknowledged that high Q HOMs are causes of various beam quality degradations. Among them most debilitating ones are so called multipass beam breakups[2,3,4] unique to recirculating linacs. Several ambitious recirculating electron accelerator projects $[5,6,7]$

\footnotetext{
*Work supported by the U. S. Department of Energy under contract DEAC05-84-ER40150, the Office of Naval Research, the Commonwealth of Virginia, and the Laser Processing Consortium.

†yunn@jlab.org
}

have recently been proposed and many more are under intensive study. Typical average beam current required for these future projects are very high indeed in the neighborhood of a few $100 \mathrm{~mA}$. At such high currents it is very likely that one of these multipass beam breakups will probably become the limiting factor of the accelerator performance. It should be noted that the multipass dipole beam breakup phenomenon has been investigated quite extensively as a part of design study of the 5-pass CEBAF superconducting accelerator and Jefferson Lab IR FEL. In both cases we had an ample safety margin (a factor of 100 for CEBAF machine and a factor of 10 for IR FEL) for beam breakups. Therefore, we didn't have to face the beam breakup directly. But the situation may be quite different in some of these new projects being proposed.

In this note we draw an attention to one more kind of multipass beam breakup phenomenon which has a potential to be very destructive if not careful. Namely a beam breakup induced by a quadrupole HOM of cavity. We present an analysis of a quadrupole beam breakup for designers of future high current projects with energy recovering linac.

\section{QUADRUPOLE WAKE}

At high energies a beam bunch passes through a cavity without its trajectory being perturbed much. Under such a circumstance it is very useful to consider integrated impulses in order to introduce wake functions to describe interactions between the beam and the beam induced electromagnetic fields. Quite generally one can derive following relations for an axially symmetric structure in the cylindrical coordinate system[8],

$$
\begin{aligned}
& ? F_{\text {parallel }} d s ? ? e I_{m} W_{m}{ }^{\prime}(z) r^{m} \cos m ? \\
& ? F_{\text {perp }} d s ? ? e I_{m} W_{m}(z) m r^{m ? 1}(\hat{r} \cos m ? ? \hat{?} \sin m ?)
\end{aligned}
$$

where the integration is for the length of the structure and $I_{m}$ is the m-th moment of the beam bunch. The function $W_{m}(z)$ is the wake function. Here $m=0$ is the monopole, $m=1$ the dipole, and $m=2$ the quadrupole wake function.

Our concern here is with $m=2$ wake functions which couple with the quadrupole moments $I_{2}$. When the transverse beam shape is not round, the beam bunch may in general possess normal and skew quadrupole moments. However, we will exclude the skew quadrupole moment 
in the following analysis. In linacs this is usually a good assumption. It should be noted that one can easily include the skew moment in the analysis when necessary.

\section{A BEAM BREAKUP ANALYSIS}

Let us consider a simple model of a 2-pass recirculating accelerator consisted of just a single cavity. Let us assume that a bunch enters the cavity with some quadrupole moments on its $1^{\text {st }}$ pass for acceleration. The bunch will excite more quadrupole HOMs to be added to already existing HOMs in the cavity. While being accelerated in the cavity the bunch will also interact with the quadrupole wakes excited by all preceded bunches. Subsequently the induced quadrupole moments will in turn add to the existing HOM fields on its $2^{\text {nd }}$ pass through the cavity. Thereby a feedback loop is closed, an exponential increase of the wakes is possible when the beam current exceeds a threshold by providing more wakes than the amount decreased which is typically quantified by the external $Q s$ of HOMs.

First, we remember that the integrated quadrupole wake potential $G_{2}(t)$ is obtained from

$$
G_{2}(t) ? \stackrel{?_{?}^{t}}{? ?} W_{2}\left(t ? t^{\prime}\right) Q_{n}\left(t^{\prime}\right) d t^{\prime}
$$

where $W_{2}(t)$ is the delta functional quadrupole wake function and $Q_{n}(t)$ is the normal component of beam bunch quadrupole moment when the bunch passes the cavity at time $t$. We note that $Q_{n}=? x^{2} ?-? y^{2} ?=?\left(?_{x^{-}} ?_{y}\right)$, where ? is the transverse emittance which we assume to be the same in $x$ and $y$ directions for the sake of simplicity.

For a single quadrupole mode with an angular frequency ?, the wake $W_{2}(t)$ is given by

$$
W_{2}(t) ? 2 \frac{c k_{\text {loss }}}{?} \exp \left(? \frac{? t}{2 Q}\right) \sin ? t
$$

for $t>0$, and $W_{2}(t)$ is equal to 0 for $t<0$ by causality. Here $k_{\text {loss }}$ is the usual loss factor for the quadrupole mode defined as follows,

$$
k_{\text {loss }} ? \frac{? R}{4 Q b^{4}}
$$

where $b$ is the beam pipe radius and $R / Q$ is to be evaluated at $b$.

Second, we notice that a bunch entering the cavity with an accumulated wake excited by all previous bunches will receive a transverse kick given by

$$
F_{x} i ? F_{y} j ? ? 2 N e^{2}(x i ? y j) \frac{G_{2}(t)}{L}
$$

where $i$ and $j$ represent unit vectors along $x$ and $y$ and $L$ is the length of cavity and $N$ is the number of electrons in a single bunch.

Consequently, we may regard that the action of the excited cavity wake on the beam is equivalent to a regular quadrupole lens with the focal length,

$$
f ? \frac{?}{2 N r_{0} G_{2}(t) L}
$$

where $r_{0}$ is the classical radius of the electron, $r_{0}=e^{2} / m c 2$ $=2.818 \times 10-13 \mathrm{~cm}$.

To proceed further without being overwhelmed by too many variables to keep track we need to simplify the problem a bit. We will assume that in the absence of quadrupole wakes the transverse cross sections of the bunch entering the cavity on its $1^{\text {st }}$ and $2^{\text {nd }}$ pass are perfectly round, which means that there will be no excitation of quadrupole wake by the design beam. Furthermore we will assume that the lattice has no $\mathrm{x}-\mathrm{y}$ coupling. Let $?_{1}=?_{x}=?_{y}$ at the $1^{\text {st }}$ pass and $?_{2}=?_{x}=?_{y}$ at the $2^{\text {nd }}$ pass for the beam at the cavity location and also let $?_{x}\left(?_{y}\right)$ be the phase advance in $x$ (and in $y$ respectively) from the cavity to the cavity for one recirculation.

Third, we are now able to calculate changes in ?'s from the design value at the $2^{\text {nd }}$ pass at the cavity location induced by the quadrupole wake following the perturbation formula worked out by D. Douglas, A. Garren and F. Dell. [9]. We obtain

$$
? ?_{x, y}(t) ? ? 2 \frac{N r_{0} G_{2}(t)}{?} ?_{1} ?_{2} \sin 2 ?_{x, y}
$$

We can now compute bunch quadrupole moment at the cavity on its $2^{\text {nd }}$ pass

$$
\begin{gathered}
Q_{n}(t) ?+2 \frac{N r_{0}}{?} ? ?_{1} ?_{2}\left(\sin 2 ?_{x} ? \sin 2 ?_{y}\right) \\
? G_{2}\left(t ? t_{?}\right) ? ?\left(t ? t_{?} ? m t_{0}\right)
\end{gathered}
$$

where $t_{r}$ is the recirculation path length in time and $t_{0}$ is the fundamental bunching period and the summation? is over all integer values of $\mathrm{m}$. ?(t) is the Dirac delta function.

Finally, we are now in a position to construct a homogeneous integral equation for the integrated wake function $G_{2}(t)$ by inserting the quadrupole moments back into the first equation of this section. We obtain 


$$
\begin{array}{rl}
G_{2}(t) ? & ? 2 \frac{N r_{0}}{?} ? ?_{1} ?_{2}\left(\sin 2 ?_{x} ? \sin 2 ?_{y}\right) \\
& ? ? W_{2}\left(t ? t^{\prime}\right) G_{2}\left(t^{\prime} ? t_{?}\right) ? ?\left(t^{\prime} ?_{?} ? m t_{0}\right) d t^{\prime}
\end{array}
$$

Searching for an exponentially growing solution for $G_{2}(t)$ we find the following expression for the threshold current, $\mathrm{I}_{\mathrm{th}}$

$$
I_{t h} ? \frac{? E \exp \left(? \frac{? t_{?}}{2 Q}\right)}{e c\left(\frac{R}{Q b^{4}}\right) Q ? ?_{1} ?_{2}\left(\sin 2 ?_{x} ? \sin 2 ?_{y}\right) \sin ? t_{?}}
$$

where the beam energy $E=? m c^{2}$. We should note that the above relationship has been derived in a perturbation expansion.

As an example, let us consider a 7-cell CEBAF cavity which is being designed for the $12 \mathrm{GeV}$ upgrade of the CEBAF accelerator. The lowest pass band start at 2568 $\mathrm{MHz}$ and the strongest quadrupole mode in that pass band has $R / Q=32.6$ ? at the beam pipe radius according to URMEL. We may get an appreciation of the relevance of the quadrupole mode beam breakup from a simple exercise with following parameters

$f=2575 \mathrm{MHz}$

$R / Q=32.6 ?$

$b=3.5 \mathrm{~cm}$

$E=100 \mathrm{MeV}$

$?=\sim 1 \mathrm{~mm}-\mathrm{mrad}$

$?_{1}, ?_{2}=\sim 10 \mathrm{~m}$

$\sin 2 ?_{x}, \sin 2 ?_{y}=\sim 1$

$\sin ? t_{r} \sim 1$

Inserting these numbers into the threshold formula we get $I_{t h}$ as a function of the loaded $Q$

$I_{t h}=2.5 ? 10^{5} / Q($ in $\mathrm{A})$

We can see that the quality factor of $2575 \mathrm{MHz}$ mode should be down to $10^{6} \sim 10^{7}$ when designing a $100 \mathrm{~mA}$ machine with only one 7-cell CEBAF cavity. It is to be reminded that this is for an accelerator with only a single 7-cell cavity of CEBAF type.

\section{CONCLUSIONS}

In high energy accelerators beam focusing is achieved mainly with quadrupoles. Consequently the beam can't be round in accelerating cavities in general and the quadrupole beam breakup instability presented in this paper is unavoidable at some point when we are pushing the current limit higher. Furthermore one should be aware that this instability will show up even after one has achieved a perfect alignment of all beamline elements. Despite many simplified assumptions made which require a future detailed study I hope that the present analysis has clearly shown the possible importance of higher order quadrupole modes in a future very high current recirculating linac and should be looked at carefully in a design stage when setting the damping requirement of HOMs, etc., for example.

\section{ACKNOWLEDGEMENTS}

We are grateful to D. Douglas for providing Reference [1] and many helpful discussions.

\section{REFERENCES}

[1] G. R. Neil et al., "Sustained Kilowatt Lasing in a FreeElectron Laser with Same-Cell Energy Recovery", Phys. Rev. Lett., 84, 662 (2000).

[2] J. J. Bisgnano and M. L. Fripp, Proc. of 1988 Linear Accelerator Conf., 388 (1988).

[3] J. J. Bisognano and R. L. Gluckstern, Proc. of 1987 Particle Accelerator Conf., 1078 (1987).

[4] B. C. Yunn, Proc. of 1991 Particle Accelerator Conf., 1785 (1991).

[5] S. Gruner et al., Rev. Sci. Inst., 73, 1402 (2002).

[6] M. W. Poole et al., Proc. of the 2002 Euro. Part. Acc. Conf., 733 (2002).

[7] E. Steffens and U. Schindler, editors, Proc. of the ERLSYN 2002 Workshop, Universitat Erlangen (2002).

[8] A. W. Chao, "Physics of Collective Beam Instabilities in High Energy Accelerators", John Wiley \& Sons, Inc. (1993).

[9] D. Douglas, A. Garren, and F. Dell, "The Collins' Scheme for the Local Correction of Chromatic Aberrations due to Insertions", LBID-911 (1984). 